\title{
Under-flap stromal bed CXL for early post-LASIK ectasia: a novel treatment technique
}

\author{
This article was published in the following Dove Press journal: \\ Clinical Ophthalmology \\ 16 December 2016 \\ Number of times this article has been viewed
}

\author{
Avi Wallerstein ${ }^{1,2}$ \\ Eser Adiguzel ${ }^{2}$ \\ Mathieu Gauvin ${ }^{1,2}$ \\ Nima Mohammad-Shahi' \\ Mark Cohen ${ }^{2,3}$ \\ 'Department of Ophthalmology, \\ Faculty of Medicine, McGill University, \\ ${ }^{2}$ LASIK MD, Montreal, ${ }^{3}$ Department \\ of Surgery, Faculty of Medicine \\ and Health Sciences, University of \\ Sherbrooke, Sherbrooke, QC, Canada
}

Correspondence: Avi Wallerstein LASIK MD, I 250 Rene-Levesque Blvd W, MD Level, Montreal, QC H3B 4W8, Canada

Tel + I 5 I49089888 ext 2273

Email awallerstein@lasikmd.com
Purpose: Collagen cross-linking (CXL) for post-laser-assisted in situ keratomileusis (LASIK) ectasia (PLE) is traditionally performed either epi-on or epi-off on the corneal surface. This study describes a novel technique in treating early PLE with under-flap CXL (ufCXL) to the stromal bed and reports on 6-month outcomes.

Patients and methods: Case series of seven patients (eight eyes) with topography-diagnosed early PLE treated with ufCXL. Inclusion criteria were early, mild PLE defined as new-onset postoperative manifest refraction cylinder $\leq 1.50 \mathrm{D}$, with new topographic inferior steepening consistent with ectasia, uncorrected distance visual acuity (UDVA) of 20/40 or better, and corrected distance visual acuity (CDVA) of 20/25 or better. Existing LASIK flap was lifted, riboflavin was applied directly to the stromal bed, flap was repositioned, and $18 \mathrm{~mW} / \mathrm{cm}^{2}$ ultraviolet light was applied for 3 minutes to the corneal surface. Post-ufCXL manifest refraction, UDVA and CDVA, corneal cylinder, $K_{\max }$, and corneal irregularity index were compared with pre-ufCXL measurements.

Results: Patients had a pre-ufCXL sphere of $0.09 \pm 0.48 \mathrm{D}$ and cylinder of $-0.78 \pm 0.49 \mathrm{D}$. At 6 months, post-ufCXL sphere $(0.06 \pm 0.8 \mathrm{D} ; P=0.89)$ and cylinder $(-1.09 \pm 0.76 \mathrm{D}, P=0.26)$ were unchanged. Cumulative post-ufCXL UDVA was unchanged, achieving 20/20, 20/30, and $20 / 40$ in $25 \%, 88 \%$, and $88 \%$, respectively, compared with $13 \%, 63 \%$, and $88 \%$ pre-ufCXL $(P=0.68)$. Post-ufCXL CDVA was unchanged $(P=0.93)$ with a gain of one line in two eyes, a loss of one line in one eye, and five eyes unchanged. The efficacy index $(P=0.76)$, safety index $(P=0.89), K_{\max }(P=0.94)$, and corneal irregularity index $(P=0.73)$ were also unchanged.

Conclusion: Preliminary results with ufCXL for early PLE are promising, demonstrating maintenance of visual accuracy, efficacy, safety, $K_{\max }$, and cylinder, with much quicker recovery times than surface CXL.

Keywords: CXL, LASIK, ufCXL, post-LASIK ectasia, cross-linking, LASIK complications, stromal bed

\section{Introduction}

Post-laser-assisted in situ keratomileusis (LASIK) ectasia (PLE) consists of progressive corneal steepening with stromal thinning due to a reduction in biomechanical stability of the cornea. ${ }^{1-4}$ Although PLE is a rare adverse outcome of laser refractive surgery, it is a significant sight-threatening complication leading to compromised visual function and patient morbidity. ${ }^{1-4}$

Corneal collagen cross-linking (CXL) halts progressive corneal ectasia by strengthening the collagen lamellae of the cornea. ${ }^{5}$ Although this treatment was only recently approved by the US Food and Drug Administration, CXL efficacy and safety have been extensively documented since 2003 in both keratoconus (KC) and PLE patients. ${ }^{5-9}$ Traditional epithelium-off CXL involves removal of the corneal epithelium, and therefore recovery is uncomfortable and long, with significant time off work, productivity loss, 
and compromised lifestyle. Furthermore, the potential for significant complications exists. ${ }^{10}$ Epithelium-on techniques have been developed to minimize recovery and complications, but have not shown the same efficacy and may not be suitable for PLE. ${ }^{11,12}$

We propose a novel, less invasive technique to treat PLE at an earlier clinical stage, before significant loss of CDVA. The method we have termed under-flap CXL (ufCXL) involves soaking the stromal bed with riboflavin, repositioning the flap, then applying ultraviolet (UV) light to the corneal surface. We hypothesize that ufCXL will halt the ectatic progression in early PLE by strengthening the corneal tissue under the flap and, in so doing, preserve existing refractive error, uncorrected distance visual acuity (UDVA), and corrected distance visual acuity (CDVA) before it worsens. By eliminating the need to remove the epithelium, it can also minimize known complications of standard epi-off CXL, minimize patient discomfort, and significantly hasten recovery time. The purpose of this study is to describe this novel technique of ufCXL in treating early PLE and to report on 6-month outcomes of the first treated eyes.

\section{Materials and methods Patient selection}

Inclusion criteria were early PLE eyes, defined as presenting with new onset of progressive manifest refraction cylinder up to $1.50 \mathrm{D}$, UDVA of 20/40 or better, CDVA of $20 / 25$ or better, and new topographic irregular astigmatism and/or inferior steepening, with under-flap stromal bed of $325 \mu \mathrm{m}$ or greater, resulting in a total corneal thickness (stromal bed + flap) of $400 \mu \mathrm{m}$ or greater. The study was approved by the Ethics Review Board of the Canadian Ophthalmic Research Centre and fulfilled all principles of the Declaration of Helsinki. Written informed consent was received from all patients.

\section{Surgical technique}

All procedures were formed using an internally-developed standardized technique for under-flap stromal CXL. The existing LASIK flap was lifted and retracted back onto a weck pillow (Merocel, Beaver-Visitec International Inc., Waltham, MA, USA) on the superior bulbar conjunctiva. Ultrasound pachymetry (Sonogage Inc., Cleveland, $\mathrm{OH}$, USA) was performed on the stromal bed. A $7 \mathrm{~mm}$ circular sponge (Corneal light shield, Beaver-Visitec International Inc.) was soaked with drops of $0.25 \%$ riboflavin solution (riboflavin dissolved in $0.87 \% \mathrm{NaCl}$; Haber's Compounding Pharmacy, Toronto, ON, Canada) and was placed on the stromal bed for a duration of 3 minutes (similar to accelerated protocols used in adjunct LASIK CXL ${ }^{13-17}$ ), with meticulous care not to expose the flap to riboflavin. The quick riboflavin soak time, using a concentration of $0.25 \%$, did not allow the retracted, exposed flap to dry out. The stromal bed was then dried, and any excess riboflavin was removed with a weck sponge. The flap was then refloated back into position, and remaining riboflavin was irrigated away. The flap was reexamined at the slit lamp and adjusted, if necessary, as per normal LASIK procedure. Accelerated UV light exposure was then performed as published elsewhere. ${ }^{13-17} \mathrm{UV}$ light (CCL-VARIO Cross-linking Radiation system; Peschke Trade GmbH, Huenenberg, Switzerland) was applied for 3 minutes at $18 \mathrm{~mW} / \mathrm{cm}^{2}$, resulting in a total irradiation dose of $3.24 \mathrm{~J} / \mathrm{cm}^{2}$ at the corneal surface. After UV treatment, the flap was again verified to be free of striae, debris, and in good position. Postoperative treatment of ufCXL eyes included the administration of Zymar four times a day for 5 days, and Pred-Forte as follows: every hour on day 1; every 2 hours on day 2; and four times a day for days 3, 4 and 5.

\section{Statistical analysis}

Data from ophthalmic examinations at pre-LASIK, preufCXL, and 1, 2, 3, 6 months, and later time points postufCXL were collected for analysis, including manifest refraction sphere, cylinder, spherical equivalent (MRSE), UDVA, CDVA, maximum keratometry $\left(K_{\max }\right)$, central pachymetry, corneal irregularity indices, presence of haze, and/or other complications at slit lamp. Keratometry and corneal irregularity indices were obtained with the Orbscan IIz system (Bausch \& Lomb, Rochester, NY, USA) at each time point. All statistical analyses were carried out in MATLAB R2016B software (Mathworks, Natick, MA, USA) using two-sample paired Student's $t$-tests (eg, to compare pre- and post-ufCXL variable means) and two-sample KolmogorovSmirnov tests (to compare pre- and post-ufCXL histogram distributions). Statistical significance was set at $P<0.05$.

\section{Results}

Data were available for eight eyes of seven patients, who underwent previous Hansatome microkeratome LASIK and presented with early PLE. The average age was $33.8 \pm 9.6$ years, mean pre-ufCXL UDVA of $0.18 \pm 0.13$ logMAR (20/30), CDVA of 0.04 $\pm 0.04 \log$ MAR (20/20-1), and cylinder of $-0.78 \pm 0.49 \mathrm{D}$. Average time to ufCXL was $30.5 \pm 13.5$ months post-LASIK, with an average follow-up time post-ufCXL of $7.6 \pm 4.1$ months. Individual patient data are available in Table 1. 


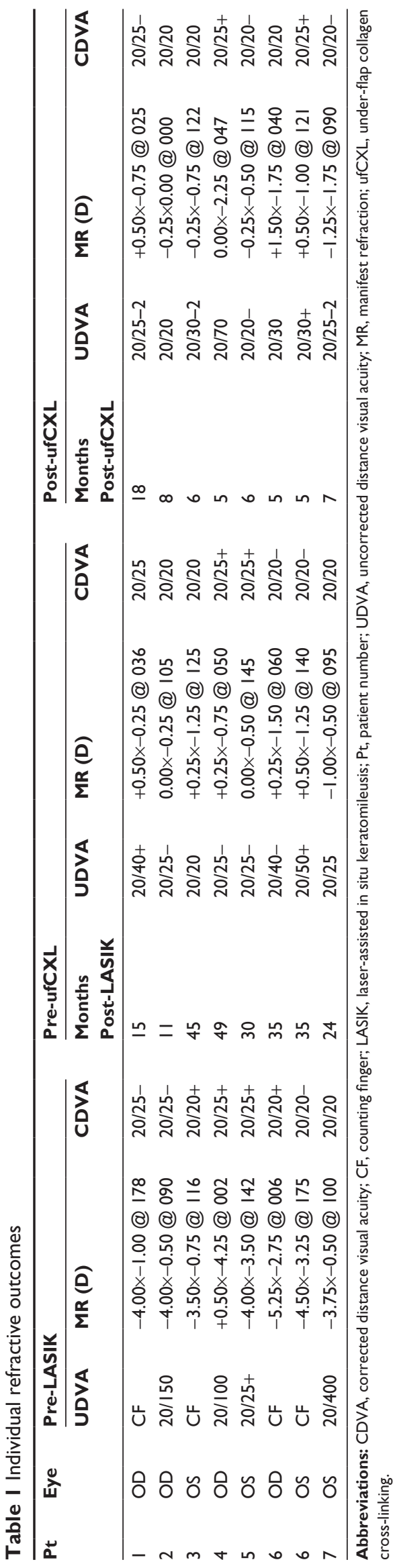

\section{Accuracy}

There was no significant difference $(P=0.44)$ in accuracy pre- to post-ufCXL, with $R^{2}$ values of 0.99 vs. 0.96 , respectively (Figure 1A). A total of $50 \%, 88 \%$ and $88 \%$ of PLE eyes were respectively within $\pm 0.25 \mathrm{D}, \pm 0.50 \mathrm{D}$, and $\pm 1.00 \mathrm{D}$ of intended correction pre-ufCXL (Figure 1B), compared with $38 \%, 50 \%$, and $75 \%$ post-ufCXL $(P=0.70)$. Moreover, $25 \%, 50 \%$, and $63 \%$ of eyes were within $0.25 \mathrm{D}, 0.50 \mathrm{D}$, and $1.00 \mathrm{D}$ of intended plano cylinder pre-ufCXL (Figure 2), compared to $13 \%, 25 \%$, and $63 \%$ post-ufCXL $(P=0.69)$, respectively.

\section{Efficacy}

At the time of diagnosis, early PLE resulted in a decreased LASIK efficacy index of $0.79 \pm 0.25$ pre-ufCXL, which was unchanged post-ufCXL $(0.83 \pm 0.31, P=0.76)$. There was a nonsignificant $(P=0.68)$ increase in the percentage of eyes achieving cumulative Snellen UDVA of 20/20 and 20/30 in post-ufCXL ( $25 \%$ and $88 \%$, respectively) vs. pre-ufCXL (13\% and $63 \%$, respectively) compared with pre-LASIK CDVA (50\% and 100\%, respectively; Figure 3A). This was apparent when comparing the difference in Snellen lines of pre-LASIK CDVA to pre-ufCXL UDVA ( $25 \%$ with three or more lines worse and $0 \%$ with one or more lines better) and pre-LASIK CDVA to post-ufCXL UDVA (12.5\% with three or more lines worse and $25 \%$ with three or more lines better, Figure 3B).

\section{Cylinder vector analysis}

Cylinder vector analysis revealed a nonsignificant trend toward overcorrection post-ufCXL compared with preufCXL (Figure 4A, Correction Index: 1.10 \pm 0.67 vs. $0.83 \pm 0.33$, respectively; $P=0.44$ ) and an increased index of success (IOS) post-ufCXL compared with pre-ufCXL (IOS: $0.86 \pm 1.12$ vs. $0.58 \pm 0.52$, respectively; $P=0.45$ ). For both pre- and post-ufCXL compared with pre-LASIK, the overall angle of error was within $-25^{\circ}$ and $+25^{\circ}$ in the majority of eyes ( $75 \%$ and $75 \%$, respectively; Figure $4 \mathrm{~B})$. There was a slight nonsignificant $(P=0.26)$ increase in cylinder magnitude 6 months post-ufCXL $(-1.09 \pm 0.76 \mathrm{D})$, compared with pre-ufCXL $(-0.78 \pm 0.49 \mathrm{D})$.

\section{Safety}

The safety index was unchanged pre- to post-ufCXL $(1.03 \pm 0.09$ vs. $1.04 \pm 0.15, P=0.89)$. Five eyes had the same Snellen CDVA post-ufCXL (Figure 5A), whereas one eye lost one line and two eyes gained one line. The cumulative 


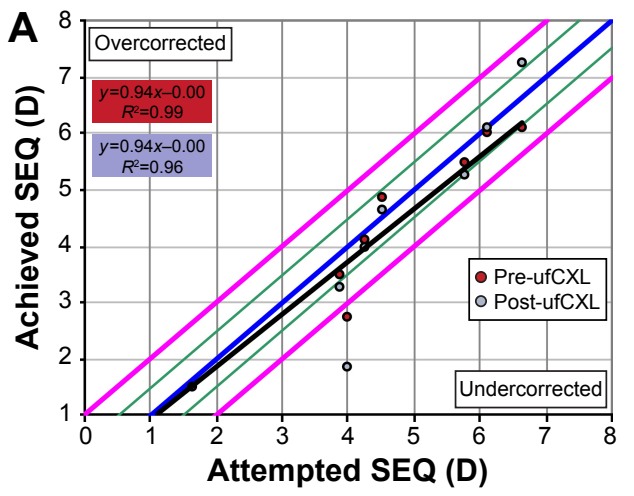

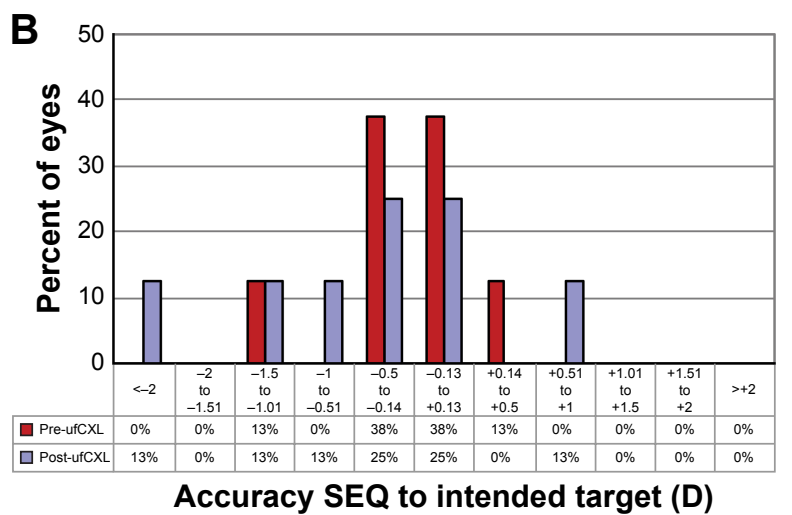

Figure I Attempted SEQ vs. achieved, pre-ufCXL compared with post-ufCXL. Blue line indicates attempted $=$ achieved, green lines indicate $+/-0.50 \mathrm{D}$ and, pink lines indicate +/- $1.00 \mathrm{D}(\mathbf{A})$. Intended target pre-ufCXL compared with post-ufCXL (B).

Abbreviations: SEQ, spherical equivalent refraction; ufCXL, under-flap collagen cross-linking.

Snellen CDVA was unchanged $(P=0.93)$ between preLASIK, pre-ufCXL, and post-ufCXL (Figure 5B).

\section{Stability}

MRSE was stable post-ufCXL at all time points examined ( $P=0.44$ at 6 months, Figure $6 \mathrm{~A})$, as was cylinder $(P=0.26$, not shown).

\section{Corneal topography}

Corneal topography showed no evidence of ectasia progression over the follow-up time period. $K_{\max }$ was stable across all time points ( $P=0.94$ at 6 months, Figure 6B). Furthermore, there was no significant difference pre- to post-ufCXL in the Orbscan IIz corneal irregularity indices within both the central $3 \mathrm{~mm}(3.21 \pm 0.87$ vs. $3.19 \pm 1.04$, respectively; $P=0.52)$ and $5 \mathrm{~mm}$ corneal areas ( $3.36 \pm 0.72$ vs. $3.31 \pm 0.70$, respectively; $P=0.73$ ).

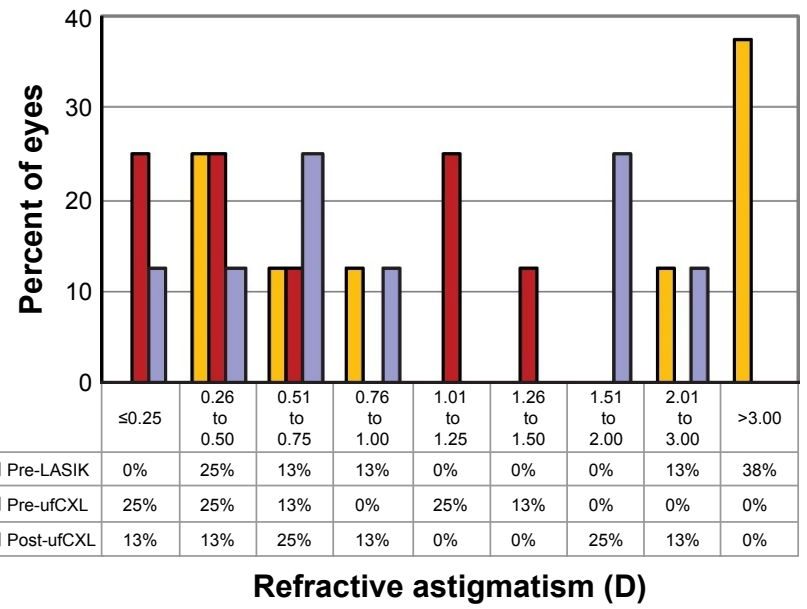

Figure 2 Refractive astigmatism accuracy pre-ufCXL and post-ufCXL compared with pre-LASIK.

Abbreviations: LASIK, laser-assisted in situ keratomileusis; ufCXL, under-flap collagen cross-linking.

\section{Complications}

One eye had mild, nonvisually significant haze that had fully resolved by the last post-ufCXL visit, and one eye had epithelial ingrowth requiring removal, which obtained $100 \%$ efficacy and safety after healing. No other complications were noted.

\section{Discussion}

CXL performed under a LASIK flap (sometimes referred to as intrastromal CXL and trade named LASIK Xtra by Avedro, Waltham, MA, USA) has been used as an adjunct with LASIK surgery. Its purpose has been prophylaxis against refractive regression in high myopia or hyperopia and to prevent ectasia in higher risk cases. ${ }^{13-15,17-22}$ The literature already documents that such ufCXL is safe ${ }^{13-15,17-22}$ with no vision loss or endothelial cell loss. To the best of our knowledge though, ufCXL has never been described as a way to prevent further progression of an early ectatic process in PLE diagnosed patients.

The reported incidence of PLE is low, varying between $0.04 \%$ and $0.6 \%$ depending on the population being studied. ${ }^{2,3}$ Given the large number of LASIK procedures performed each year, even a low incidence of PLE is significant and warrants efforts to reduce its occurrence and also to improve on current available treatments. In the current study, using ufCXL we present a novel, less invasive technique than traditional epi-off CXL to treat PLE at an early clinical stage, before significant loss of CDVA.

We treated seven patients (eight eyes), with CXL under the LASIK flap (ufCXL), who developed clear signs of early PLE. We used the same parameters (riboflavin concentration and UV light dose) as previously described accelerated protocols for adjunct LASIK CXL. This technique leaves an intact epithelium and therefore has the potential to minimize 
A

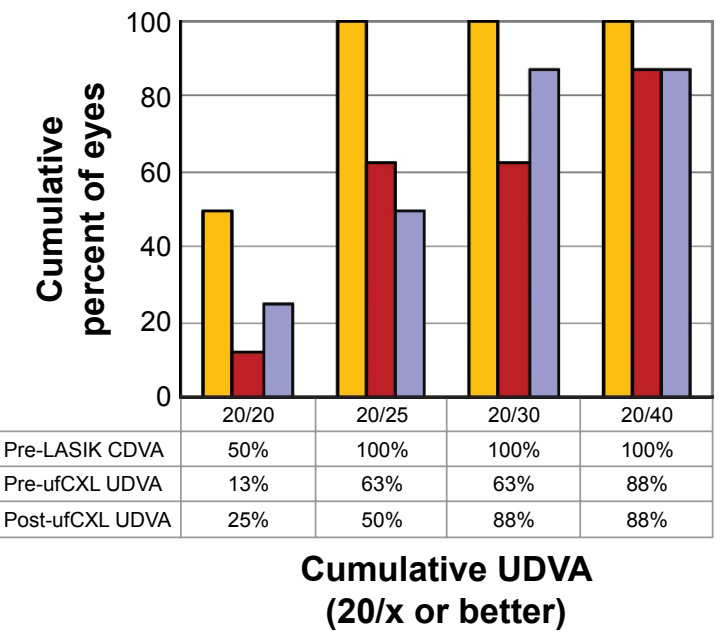

B

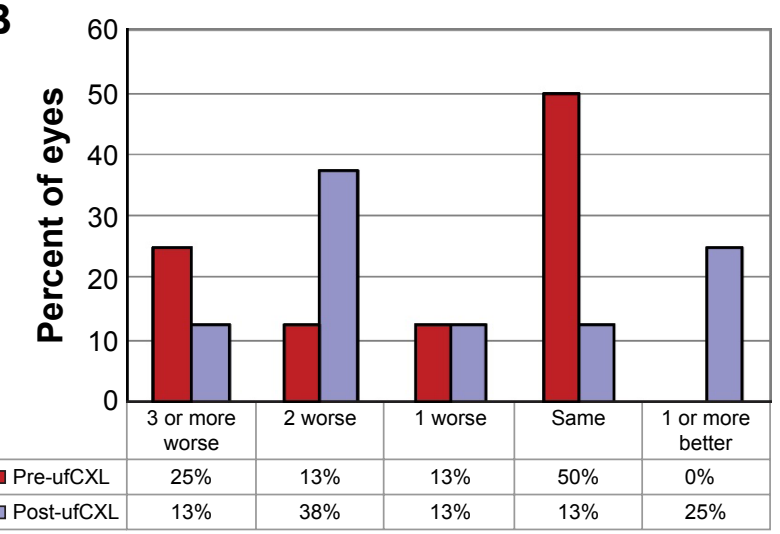

Difference between UDVA and CDVA

Figure 3 Cumulative Snellen UDVA pre- and post-ufCXL compared with pre-LASIK CDVA (A). Difference in Snellen lines of UDVA pre- and post-ufCXL compared with pre-LASIK CDVA (B).

Abbreviations: CDVA, corrected distance visual acuity; LASIK, laser-assisted in situ keratomileusis; UDVA, uncorrected distance visual acuity; ufCXL, under-flap collagen cross-linking.
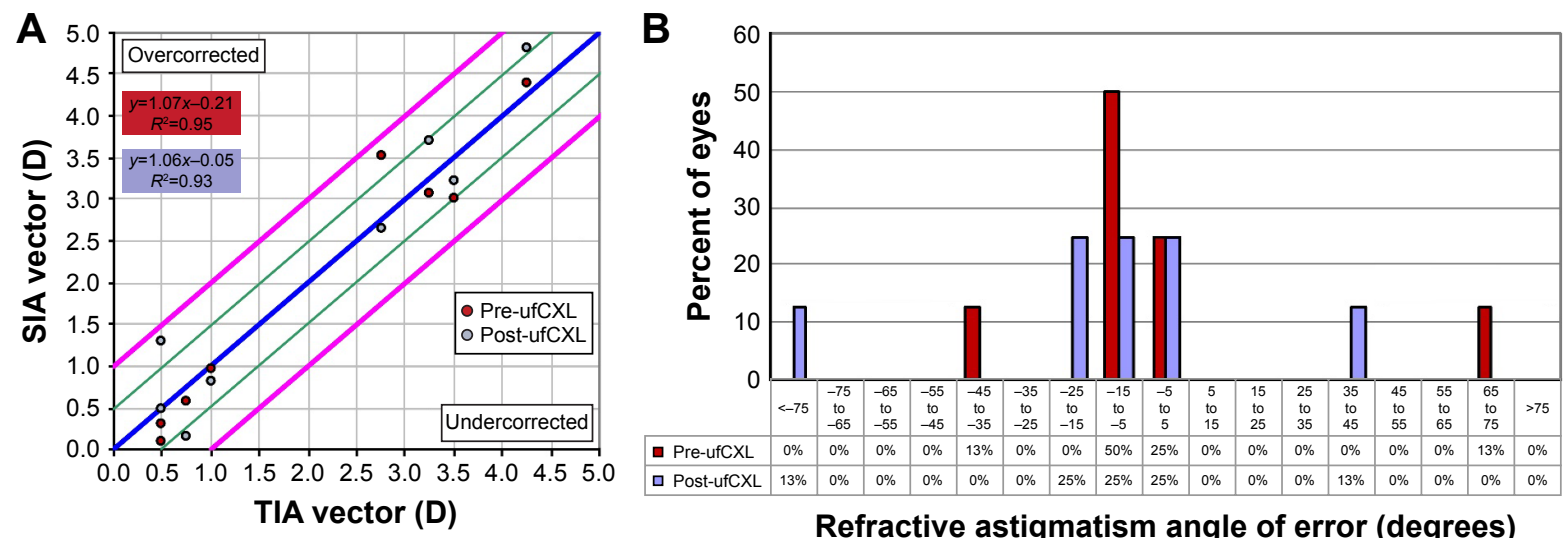

Refractive astigmatism angle of error (degrees)

Figure 4 Pre-LASIK TIA vector vs. SIA vector for pre- and post-ufCXL. Blue line indicates TIA = SIA, green lines indicate +/- 0.50D, pink lines indicate +/- I.00D (A) Distribution of the refractive astigmatism angle of error for pre- and post-ufCXL (B).

Abbreviations: LASIK, laser-assisted in situ keratomileusis; SIA, surgically induced astigmatism; TIA, target-induced astigmatism; ufCXL, under-flap collagen cross-linking.

A

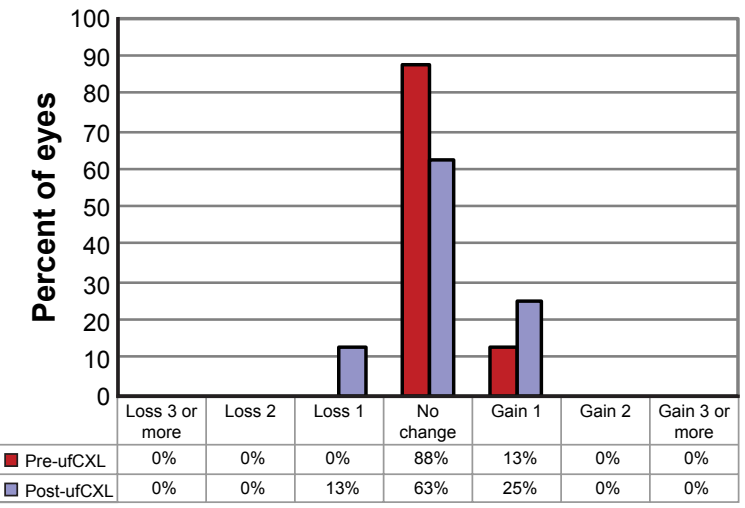

Change in lines of CDVA

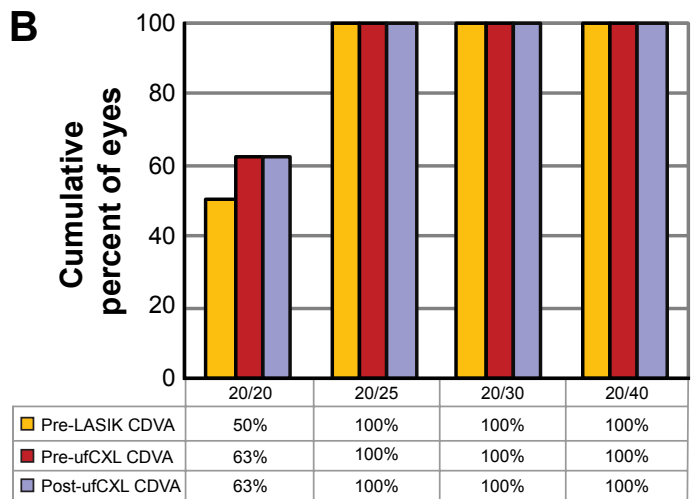

Cumulative CDVA (20/x or better)

Figure 5 Change in Snellen lines of CDVA pre- and post-ufCXL compared with pre-LASIK CDVA (A). Cumulative Snellen CDVA pre- and post-ufCXL compared with pre-LASIK CDVA (B).

Abbreviations: CDVA, corrected distance visual acuity; LASIK, laser-assisted in situ keratomileusis; ufCXL, under-flap collagen cross-linking. 
A

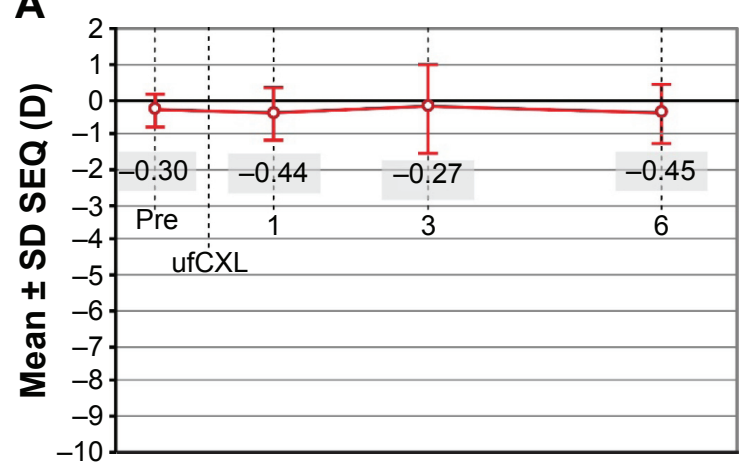

Time after ufCXL (months)
B

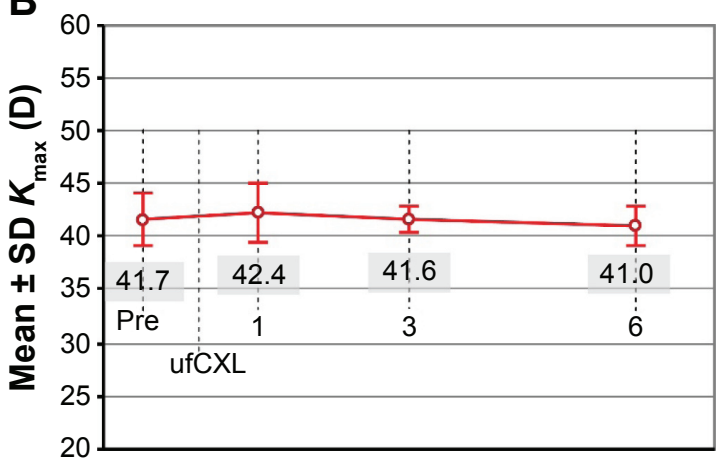

Time after ufCXL (months)

Figure 6 SEQ from pre- to post-ufCXL (A). Stability of $K_{\max }$ from pre- to post-ufCXL (B).

Abbreviations: SEQ, stability of spherical equivalent; SD, standard deviation; ufCXL, under-flap collagen cross-linking.

known complications of corneal surgery and those of epi-off CXL, such as delayed epithelial healing and slow recovery, keratitis, dry eye, haze, loss of visual acuity, induced refractive error, and more. ${ }^{23-27}$ None of the eyes presented here experienced any of these complications, including any significant haze. Epithelial ingrowth was seen in one eye. It is a potential complication of flap re-lifting, and needs to be monitored and dealt with as with all LASIK flap relifts.

To our knowledge, the only report similar to this technique for early PLE is the case of a 28-year-old female who presented 4 months post-LASIK with unilateral inferior corneal steepening and decreased visual acuity. ${ }^{28}$ The one eye was treated with what was termed intrastromal CXL, and ended up asymptomatic with 20/20 acuity and no sign of disease progression on corneal topography after 1 year. ${ }^{28}$ In contrast to our study, the flap was only opened, and riboflavin was injected between the flap and the stroma bed. The flap was, therefore, also soaked in riboflavin and was cross-linked. In our case series, the flap was fully retracted, and riboflavin was administered only to the stromal bed, and after removal of any excess solution, the flap was repositioned. UV light was then administered to the surface of the cornea, thereby crosslinking the riboflavin-soaked stromal bed under the flap. This ufCXL technique allows strengthening and stiffening of the underlying cornea rather than the flap, which in LASIK surgery provides little biomechanical strength to the cornea. ${ }^{29}$

Our inclusion criteria were topographic and refractive signs consistent with early PLE. In this way, any ectatic progression could be halted at a point where visual acuity was not yet significantly compromised, and good vision could be preserved after treatment. Our preliminary results demonstrate the maintenance of visual accuracy, efficacy, and safety at 6 months post-ufCXL, with quicker recovery times than traditional epi-off CXL. Stability was also maintained at 6 months, with no evidence of progressive ectasia based on refraction, vision, $K_{\max }$, topographic cylinder, and corneal irregularity indices.

Post-LASIK patients are often not followed up routinely with regularly scheduled postoperative topographies. If they develop ectasia, they present with moderate to advanced disease once they complain of poor vision and seek an enhancement. As well, the few PLE patients who do present early, with only topographic signs of ectasia yet minimal refractive changes and good UDVA and CDVA, are often not immediately treated. Both surgeons and patients are reluctant to treat (or be treated) with standard epi-off CXL at these earliest PLE stages due to the risk of CXL complications, the potential for the procedure itself to worsen vision, the long recovery period, and the surgeon's reflex to document progression as with keratoconus, before embarking on a relatively invasive intervention. ${ }^{23-27}$ As a result, PLE patients tend to be treated months to years after early PLE signs, with their CDVA already compromised. ${ }^{7,30,31}$

While it is indeed difficult to determine which new cases of early PLE will be progressive and to what extent, and without a control group in this study, we cannot be definitive that it is the intervention that halted progression, we do know that a large number of PLE cases lead to significant visual loss with difficult rehabilitation affecting quality of life. We also know that a high percentage of KC patients progress. Having a LASIK flap that destabilizes a cornea may cause a greater percentage of PLE patients to progress. A treatment that poses little risk to safety or visual downside allows immediate intervention once a diagnosis of PLE is made, in order to preserve vision before significant loss of CDVA occurs and before significant irregular astigmatism contributes to complicated visual rehabilitation. With regular monitoring, if progression were detected, ufCXL could 
be repeated or epi-off CXL initiated at that time. Although the current follow-up was only 6 months (with one eye followed for 18 months), if stability maintains long term as with epi-off CXL treatment, then treating early PLE eyes with ufCXL would outweigh the risks of disease progression followed by treating with the epi-off technique. It would also change the standard of care for post-LASIK surgery, requiring consistent long-term follow-up with regularly scheduled topographies, looking for early signs of ectasia that could benefit from early ufCXL in order to preserve vision.

\section{Limitations}

While this preliminary experimental case series provides first data and guidelines for this novel PLE treatment strategy, it has limitations. Our small sample of patients (eight eyes) reflects the low incidence of PLE and potentially limits our statistical power. The latter might have prevented us from detecting subtle trends in the data, such as the slight increase in post-ufCXL cylinder documented herein. In addition, we did not perform optical coherence tomography imaging to assess the depth of the CXL demarcation line, thought to indicate the extent of CXL effect. We recognize that the flap absorbs energy and decreases the total UV radiation dose. Notwithstanding, we used the same protocols as with adjunct LASIK CXL which showed good effect. ${ }^{13-17}$ Even smaller total irradiation doses demonstrated sufficient UV light absorption with post-treatment demarcation lines as deep as $280 \mu \mathrm{m}$. Considering that the clinical scenario in this study is PLE with only under-flap tissue being cross linked, higher total doses may be required. The amount of total energy delivered to the stromal bed and the actual amount of cross-linked tissue necessary to halt progression in early PLE needs to be elucidated with further studies, and reliable in vivo biomechanical measurements would prove useful. While in vivo confocal microscopy revealed no endothelial cell loss 12 months after adjunct CXL with LASIK, ${ }^{13}$ endothelial cell count was not performed herein and should therefore be investigated in future ufCXL studies.

\section{Conclusion}

This study presents the first case series of ufCXL for early PLE. Our preliminary 6-month results are promising, demonstrating maintenance of visual accuracy, efficacy, and safety, with no evidence of progressive corneal ectasia based on vision, refraction, $K_{\max }$, topographic cylinder, and corneal irregularity indices. None of the reported significant complications associated with epi-off CXL were seen. While our study is limited to a small sample of eyes, reflecting the low-incidence of
PLE, it suggests that ufCXL has the potential to be a safe and effective method of preserving visual function by halting PLE progression early, prior to its detrimental effects. Randomized case-control studies with more patients and longer follow-up times are required to further validate these findings.

\section{Disclosure}

The authors report no conflicts of interest in this work.

\section{References}

1. Pallikaris IG, Kymionis GD, Astyrakakis NI. Corneal ectasia induced by laser in situ keratomileusis. J Cataract Refract Surg. 2001;27(11): 1796-1802.

2. Santhiago MR, Giacomin NT, Smadja D, Bechara SJ. Ectasia risk factors in refractive surgery. Clin Ophthalmol. 2016;10:713-720.

3. Seiler T, Koufala K, Richter G. Iatrogenic keratectasia after laser in situ keratomileusis. J Refract Surg. 1998;14(3):312-317.

4. Spadea L, Cantera E, Cortes M, Conocchia NE, Stewart CW. Corneal ectasia after myopic laser in situ keratomileusis: a long-term study. Clin Ophthalmol. 2012;6:1801-1813.

5. Wollensak G, Spoerl E, Seiler T. Riboflavin/ultraviolet-a-induced collagen crosslinking for the treatment of keratoconus. Am J Ophthalmol. 2003;135(5):620-627.

6. Brooks NO, Greenstein S, Fry K, Hersh PS. Patient subjective visual function after corneal collagen crosslinking for keratoconus and corneal ectasia. J Cataract Refract Surg. 2012;38(4):615-619.

7. Hafezi F, Kanellopoulos J, Wiltfang R, Seiler T. Corneal collagen crosslinking with riboflavin and ultraviolet $\mathrm{A}$ to treat induced keratectasia after laser in situ keratomileusis. J Cataract Refract Surg. 2007;33(12):2035-2040.

8. Hersh PS, Greenstein SA, Fry KL. Corneal collagen crosslinking for keratoconus and corneal ectasia: one-year results. $J$ Cataract Refract Surg. 2011;37(1):149-160.

9. Kanellopoulos AJ. Comparison of sequential vs same-day simultaneous collagen cross-linking and topography-guided PRK for treatment of keratoconus. J Refract Surg. 2009;25(9):S812-S818.

10. Dhawan S, Rao K, Natrajan S. Complications of corneal collagen crosslinking. J Ophthalmol. 2011;2011:869015.

11. Caporossi A, Mazzotta C, Paradiso AL, Baiocchi S, Marigliani D, Caporossi T. Transepithelial corneal collagen crosslinking for progressive keratoconus: 24-month clinical results. J Cataract Refract Surg. 2013;39(8):1157-1163.

12. Wollensak G, Iomdina E. Biomechanical and histological changes after corneal crosslinking with and without epithelial debridement. J Cataract Refract Surg. 2009;35(3):540-546.

13. Tomita M, Yoshida Y, Yamamoto Y, Mita M, Waring Gt. In vivo confocal laser microscopy of morphologic changes after simultaneous LASIK and accelerated collagen crosslinking for myopia: one-year results. J Cataract Refract Surg. 2014;40(6):981-990.

14. Nguyen MK, Chuck RS. Corneal collagen cross-linking in the stabilization of PRK, LASIK, thermal keratoplasty, and orthokeratology. Curr Opin Ophthalmol. 2013;24(4):291-295.

15. Aslanides IM, Mukherjee AN. Adjuvant corneal crosslinking to prevent hyperopic LASIK regression. Clin Ophthalmol. 2013;7:637-641.

16. Tamayo GE. Predictable visual outcomes with accelerated corneal cross-linking concurrent with laser in situ keratomileusis. J Cataract Refract Surg. 2012;38(12):2206; author reply 2207-2208.

17. Celik HU, Alagoz N, Yildirim Y, et al. Accelerated corneal crosslinking concurrent with laser in situ keratomileusis. J Cataract Refract Surg. 2012;38(8):1424-1431.

18. Kanellopoulos AJ, Asimellis G. Epithelial remodeling after femtosecond laser-assisted high myopic LASIK: comparison of stand-alone with LASIK combined with prophylactic high-fluence cross-linking. Cornea. 2014;33(5):463-469. 
19. Kanellopoulos AJ, Kahn J. Topography-guided hyperopic LASIK with and without high irradiance collagen cross-linking: initial comparative clinical findings in a contralateral eye study of 34 consecutive patients. $J$ Refract Surg. 2012;28 (Suppl 11):S837-S840.

20. Kymionis GD, Grentzelos MA, Portaliou DM, Kankariya VP, Randleman JB. Corneal collagen cross-linking (CXL) combined with refractive procedures for the treatment of corneal ectatic disorders: CXL plus. J Refract Surg. 2014;30(8):566-576.

21. Mazzotta C, Balestrazzi A, Traversi C, Caragiuli S, Caporossi A. In vivo confocal microscopy report after lasik with sequential accelerated corneal collagen cross-linking treatment. Case Rep Ophthalmol. 2014; 5(1):125-131.

22. Kanellopoulos AJ. Long-term safety and efficacy follow-up of prophylactic higher fluence collagen cross-linking in high myopic laser-assisted in situ keratomileusis. Clin Ophthalmol. 2012;6:1125-1130.

23. Caporossi A, Mazzotta C, Baiocchi S, Caporossi T. Long-term results of riboflavin ultraviolet a corneal collagen cross-linking for keratoconus in Italy: the Siena eye cross study. Am J Ophthalmol. 2010;149(4): 585-593.

24. Kymionis GD, Bouzoukis DI, Diakonis VF, Portaliou DM, Pallikaris AI, Yoo SH. Diffuse lamellar keratitis after corneal crosslinking in a patient with post-laser in situ keratomileusis corneal ectasia. J Cataract Refract Surg. 2007;33(12):2135-2137.
25. Kymionis GD, Portaliou DM, Bouzoukis DI, et al. Herpetic keratitis with iritis after corneal crosslinking with riboflavin and ultraviolet A for keratoconus. J Cataract Refract Surg. 2007;33(11):1982-1984.

26. Pollhammer M, Cursiefen C. Bacterial keratitis early after corneal crosslinking with riboflavin and ultraviolet-A. J Cataract Refract Surg. 2009;35(3):588-589.

27. Raiskup-Wolf F, Hoyer A, Spoerl E, Pillunat LE. Collagen crosslinking with riboflavin and ultraviolet-A light in keratoconus: long-term results. J Cataract Refract Surg. 2008;34(5):796-801.

28. Moscovici BK, Campos M. Intrastromal crosslinking in post-LASIK ectasia. Arq Bras Oftalmol. 2014;77(3):191-192.

29. Eleftheriadis H, Prandi B, Diaz-Rato A, Morcillo M, Sabater JB. The effect of flap thickness on the visual and refractive outcome of myopic laser in situ keratomileusis. Eye (Lond). 2005;19(12):1290-1296.

30. Geggel HS, Talley AR. Delayed onset keratectasia following laser in situ keratomileusis. J Cataract Refract Surg. 1999;25(4):582-586.

31. Rao SN, Epstein RJ. Early onset ectasia following laser in situ keratomileusus: case report and literature review. J Refract Surg. 2002;18(2): $177-184$.
Clinical Ophthalmology

\section{Publish your work in this journal}

Clinical Ophthalmology is an international, peer-reviewed journal covering all subspecialties within ophthalmology. Key topics include: Optometry; Visual science; Pharmacology and drug therapy in eye diseases; Basic Sciences; Primary and Secondary eye care; Patient Safety and Quality of Care Improvements. This journal is indexed on

\section{Dovepress}

PubMed Central and CAS, and is the official journal of The Society of Clinical Ophthalmology (SCO). The manuscript management system is completely online and includes a very quick and fair peer-review system, which is all easy to use. Visit http://www.dovepress.com/ testimonials.php to read real quotes from published authors. 